\title{
Moving towards optimal therapy in paediatric rheumatology
}

\section{Jay Mehta}

I read with great interest the Perspectives article by Dr Tim Niehues (Optimizing treatment in paediatric rheumatology - lessons from oncology. Nat. Rev. Rheumatol. 11, 493-499; 2015) ${ }^{1}$ published in April 2015. Dr Niehues is correct in his assertion that some paediatric rheumatic diseases are as aggressive and fatal as childhood malignancies. I would point to juvenile systemic lupus erythematosus (SLE) and macrophage activation syndrome as two common rheumatic diseases which are potentially fatal if untreated. I agree with Dr Niehues that children with a rheumatic disease would benefit greatly from enrolment in treatment-optimizing study protocols such as those used in paediatric oncology. Indeed, the reduction in mortality from paediatric cancer is one of the greatest achievements in child health of the past few years.

The Childhood Arthritis and Rheumatology Research Alliance (CARRA), of which Dr Niehues makes brief mention, was formed to enrol every child with a rheumatic disease in a research study as one of its intents. A major focus of CARRA resources has been the development of consensus treatment plans (CTPs) for the major rheumatic diseases, including polyarticular juvenile idiopathic arthritis (JIA) $)^{2}$, systemic JIA ${ }^{3}$, lupus nephritis $^{4}$, juvenile dermatomyositis ${ }^{5}$ and juvenile localized scleroderma ${ }^{6}$. These CTPs have been developed through a rigorous process that includes surveys, expert opinion and face-to-face meetings of paediatric rheumatologists to achieve standardization and consensus and, consequently, increase the power of observational studies. Each CTP includes information on timing, dosage and data collection parameters and schedules. Many of the CTPs include specimen collection for future pharmacogenomic analyses and other translational studies. These CTPs will be implemented through CARRA Registry sites, enabling observational, comparative and effectiveness studies. Through an iterative process, the best treatments for these diseases will be identified. Results from pilot studies ${ }^{7,8}$ have suggested that CARRA sites are highly effective at enrolling patients into CTPs, suggesting that we will be able to approach Dr Niehues's goal of standardization in paediatric rheumatology research. The process for creating CTPs has begun for paediatric antineutrophil cytoplasmic autoantibody (ANCA)-associated vasculitis and chronic noninfectious osteomyelitis, two rheumatic diseases with potentially devastating consequences.

A major difference between CARRA's approach and that used in paediatric oncology is that the paediatric rheumatology CTPs are observational rather than randomized clinical trials. Although enrolment of each child with a rheumatic disease into a clinical trial is a goal that the paediatric rheumatologist community should be striving towards, as Dr Niehues states, this process took decades and dedicated
NIH funding for the paediatric oncology group. Through CARRA, and other organizations such as the Paediatric Rheumatology International Trials Organization (PRINTO), we are well on our way to achieving that goal.

Jay Mehta is at the Division of Rheumatology, Children's Hospital of Philadelphia, 3401 Civic Center Boulevard, Philadelphia, Pennsylvania 19147, USA. mehtaj@email.chop.edu

doi:10.1038/nrrheum.2016.21 Published online 25 Feb 2016

1. Niehues, T. Optimizing treatment in paediatric rheumatology - lessons from oncology. Nat. Rev. Rheumatol. 11, 493-499 (2015).

2. Ringold, S. et al. Childhood Arthritis and Rheumatology Research Alliance consensus treatment plans for new-onset polyarticular juvenile idiopathic arthritis. Arthritis Care Res. (Hoboken) 66, 1063-1072 (2014).

3. DeWitt, E. M. et al. Consensus treatment plans for new-onset systemic juvenile idiopathic arthritis. Arthritis Care Res. (Hoboken) 64, 1001-1010 (2012).

4. Mina, R. et al. Consensus treatment plans for induction therapy of newly diagnosed proliferative lupus nephritis in juvenile systemic lupus erythematosus. Arthritis Care Res. (Hoboken) 64, 375-383 (2012)

5. Huber, A. M. et al. Consensus treatments for moderate juvenile dermatomyositis: beyond the first two months. Results of the second Childhood Arthritis and Rheumatology Research Alliance consensus conference. Arthritis Care Res. (Hoboken) 64, 546-553 (2012).

6. Li, S. C. et al. Development of consensus treatment plans for juvenile localized scleroderma: a roadmap toward comparative effectiveness studies in juvenile localized scleroderma. Arthritis Care Res. (Hoboken) 64, 1175-1185 (2012).

7. Kimura, Y. et al. Results from the Childhood Arthritis and Rheumatology Research Alliance systemic JIA consensus treatment plans pilot study. Arthritis Rheumatol. 67 (Suppl. 10), 959 (2015).

8. Li, S. C. et al. A pilot study of juvenile localized scleroderma (jLS) consensus treatment plans. Arthritis Rheum. 65 (Suppl. 10), 2177 (2013).

Acknowledgements

Dr Mehta would like to acknowledge the Arthritis Foundation, the American College of Rheumatology, and the National Institute of Arthritis and Musculoskeletal and Skin Diseases, who have generously supported development of the Childhood Arthritis and Rheumatology Research Alliance (CARRA) Consensus Treatment Protocols.

Competing interests statement Dr Mehta receives salary support from CARRA. 